\title{
Opinión
}

\section{Síndrome metabólico: percepción de enfermedad y falta de adherencia a la prescripción médica}

\author{
(Metabolic syndrome: disease perception and \\ lack of adherence to medical advice)
}

José A. Martínez-Ortiz

\begin{abstract}
Resumen:
Se presenta el caso de una mujer de 52 años sedentaria y con sobrepeso (índice de masa corporal $=28.3$ ), que tiene, según ATP-III, las 5 características del síndrome metabólico: diabetes tipo 2 (glicemia en ayunas $=294 \mathrm{mg} / \mathrm{dL}$ ), triacilglicerol $=162 \mathrm{mg} / \mathrm{dL}$, HDL-colesterol $=44 \mathrm{mg} / \mathrm{dL}$ y obesidad central (perímetro de la cintura $=90 \mathrm{~cm}$ ). La diabetes tipo 2 le fue diagnosticada hace 17 años, recibe medicamento para la hipertensión desde hace 7 años y presenta hipercolesterolemia (colesterol total $=283 \mathrm{mg} / \mathrm{dL}$ ), para lo que no recibe tratamiento farmacológico. A pesar de su condición, la paciente se considera saludable lo cual le impide modificar su estilo de vida y mejorar la calidad de vida lo que aumenta el costo de su tratamiento médico para el sistema de seguridad social.
\end{abstract}

Descriptores: Diabetes tipo 2, síndrome metabólico, hipercoleterolemia

La evidencia disponible ${ }^{1-6}$ indica que la actividad física produce mejoras en la salud y en la calidad de vida en personas diabéticas, hipertensas y con sobrepeso. Existe una correspondencia entre la inactividad física ${ }^{7}$ y la diabetes ${ }^{8}$ y ambas tienen una incidencia negativa en la salud de los adultos costarricenses. Sin embargo, es difícil explicar por qué personas con estas patologías no adoptan estilos de vida más saludables, que incluyen un incremento en la actividad física que realizan y en la dieta.

Paciente femenina postmenopausica de 52 años, multípara, cuyos tres primeros embarazos (el primero a los 16 años) resultaron en pérdida, y los dos últimos son

Catedrático, Universidad de Costa Rica

Abreviaturas: ATP, adult treatment panel;ECV, enfermedad cardiovascular; HDL, lipoproteína de alta densidad; IMC, índice de masa corporal; LDL, lipoproteína de baja densidad.

Correspondencia: José A. Martínez-Ortiz

Correo electrónico: jamartinezortiz@yahoo.com

ISSN 0001-6002/2008/50/4/248-250

Acta Médica Costarricense, $(2008$

Colegio de Médicos y Cirujanos hombres saludables; diagnosticada con diabetes tipo 2 desde hace 17 años e hipertensión hace 7 años, que padece de pie diabético, retinopatía y dislipidemia.

Se diseñaron cuestionarios específicos para guiar las múltiples entrevistas que se le efectuaron y para valorar la calidad de vida de la paciente. Se fueron desarrollando nuevos cuestionarios para valorar diferentes aspectos del estado de salud y actividad física de la paciente estudiada, a medida que se progresó en el análisis del caso. Se aplicaron los cuestionarios desarrollados para que la paciente autoevaluara su estado de salud, sus factores de riesgo, morbilidades y nivel de actividad física. Se entrevistó a la persona en varias ocasiones; se comentaron las respuestas a los diferentes cuestionarios.

Se analizaron las posibles razones por las que la paciente no adopta prácticas de actividad física regular que ayudaran a mejorar su calidad de vida, y se presentan recomendaciones de cómo podría el sujeto adoptarlas.

La sujeto estudiada padece, de acuerdo con el criterio de ATP III, ${ }^{9}$ el síndrome metabólico en todos los cinco parámetros de evaluación: perímetro de cintura $>88 \mathrm{~cm}$, diabetes tipo 2 (tratada con insulina inyectada y metformina), hipertensa (determinado por la toma de medicamento, amlodipina, para su control), triglicéridos $>150 \mathrm{mg} / \mathrm{dL}$ y HDL-colesterol $<50 \mathrm{mg} / \mathrm{dL}$; lo cual aumenta su riesgo de morbilidad y mortalidad, aspecto que se incrementa al considerar que su diabetes no está controlada y ha permanecido así desde que le fuera diagnosticada hace 17 años. Los primeros 7 años después que le fuera diagnosticada, diabetes, fue tratada con dieta y ejercicio; los siguientes dos años con metformina, y a partir del $9^{\circ}$ año se ha tratado con insulina inyectada y metformina aunque desde que recuerda la paciente, la glicemia en ayunas siempre ha superado los $200 \mathrm{mg} / \mathrm{dL}$. No se han determinado los niveles de hemoglobina glicosilada. Adicionalmente, hace 10 meses le fue diagnosticado hígado graso, que según describe la paciente, le indicaron que era una condición "típica de diabéticos" y desde hace 3 años se le diagnosticó retinopatía diabética. 
En el cuadro se resumen las características de la paciente y en la figura se presenta la distribución del tiempo semanal que confirma su estilo de vida sedentario, lo cual se acentúa por sus hábitos de alimentación que agravan su condición. El perímetro de la cintura de la paciente fue medida por la médico general; los valores de colesterol total, HDLcolesterol, triglicéridos y glicemia fueron transcritos por la médico de los resultados realizados en el laboratorio clínico de la Caja Costarricense de Seguro Social.

\section{Discusión}

La paciente se consideraba "una persona enfermiza": a la edad de 7 años padeció de fiebre reumática, que según describe le impidió caminar durante 12 meses, debiendo ser llevada a la escuela en carreta por sus hermanos. Contradictoriamente, la paciente afirma tener en la actualidad una mejor salud que personas de su misma edad y género. La diabetes que sufre desde hace 17 años la describe como una "enfermedad silenciosa", lo que la hace olvidar que la padece; a pesar de lo anterior, manifiesta su deseo de vivir muchos años. Hasta hace 10 meses comía lo que se le

\section{Cuadro 1. Características del sujeto de estudio}

\begin{tabular}{|c|c|}
\hline Parámetro & Valor \\
\hline Género & Femenino \\
\hline Edad, años & 52 \\
\hline Diagnóstico de diabetes tipo 2, años (hace) & 17 \\
\hline Diagnóstico hipertensión, años (hace) & 7 \\
\hline Peso, kg & 62 \\
\hline Talla, cm & 148 \\
\hline Perímetro de cintura, $\mathrm{cm}$ & 90 \\
\hline Glicemia*», mg/dL & 294 \\
\hline Colesterol total ${ }^{*} \|, \mathrm{mg} / \mathrm{dL}$ & 283 \\
\hline HDL colesterol ${ }^{*}, \|, \mathrm{mg} / \mathrm{dL}$ & 44 \\
\hline Triacilglicerol ${ }^{*} \|, \mathrm{mg} / \mathrm{dL}$ & 162 \\
\hline LDL colesterol ${ }^{*, \dagger}, \mathrm{mg} / \mathrm{dL}$ & 207 \\
\hline Presión arterial sistólica ${ }^{\ddagger}, \mathrm{mmHg}$ & 131 \\
\hline Presión arterial diastólica ${ }^{\ddagger}, \mathrm{mmHg}$ & 75 \\
\hline \multicolumn{2}{|c|}{ 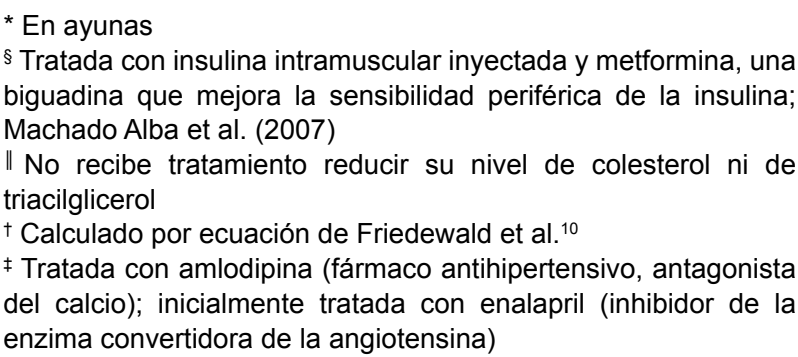 } \\
\hline
\end{tabular}

antojaba aunque confiesa que seguía la prescripción médica de caminar 2 veces por semana durante 20 minutos, que le fuera dada en el momento cuando se le diagnosticó la diabetes tipo 2, hace 17 años. La prognosis sobre el riesgo de sufrir complicaciones propias de su problema de salud es alta. El riesgo de esta paciente de padecer enfermedad cardiovascular (ECV), enfermedad vascular periférica, daño renal, accidente cerebro vascular, retinopatía diabética (que le fue diagnosticada hace 3 años) y muerte, es elevada, lo cual contrasta con su falta de conciencia para aquilatar su precario estado de salud y elevado riesgo de morbilidad y mortalidad, además de la renuencia de su parte a aceptar que un aumento en la actividad física mejoraría el control de su enfermedad. La paciente justifica su estilo de vida, aunque sigue el tratamiento para intentar, infructuosamente, mantener bajo control el nivel de glicemia. Contrariamente a lo que sucede con el nivel de glicemia, la hipertensión que sufre se mantiene controlada con el medicamento que recibe y cuyo tratamiento sigue. Este caso constituye todo un dilema ya que la paciente, a pesar de que toma medicamentos para el control de la glicemia y la hipertensión, no se considera una persona enferma y lleva un estilo de vida no adecuado para su situación, que ni siquiera intenta modificar. Este comportamiento podría ser común en pacientes con diabetes u otras enfermedades crónicas que podrían ser interpretadas por los pacientes como "silenciosas" en las que la vida no peligra de manera inminente. Esta característica hace posible que el afectado pueda rehusar aceptar su enfermedad, a pesar de que la primera línea de tratamiento consiste en cambios en el estilo de vida, ${ }^{11-13}$ que permitan llevar vida de calidad pudiendo realizar autónomamente las actividades del diario vivir, sin padecer en los últimos años de vida de incapacidad y tener una terapia farmacológica de alto costo para el sistema de seguridad social y para la sociedad.

La progenitora de la paciente, diabética, murió a los 67 años después amputársele la pierna derecha, como consecuencia de la diabetes que padecía; de los 17 hermanos

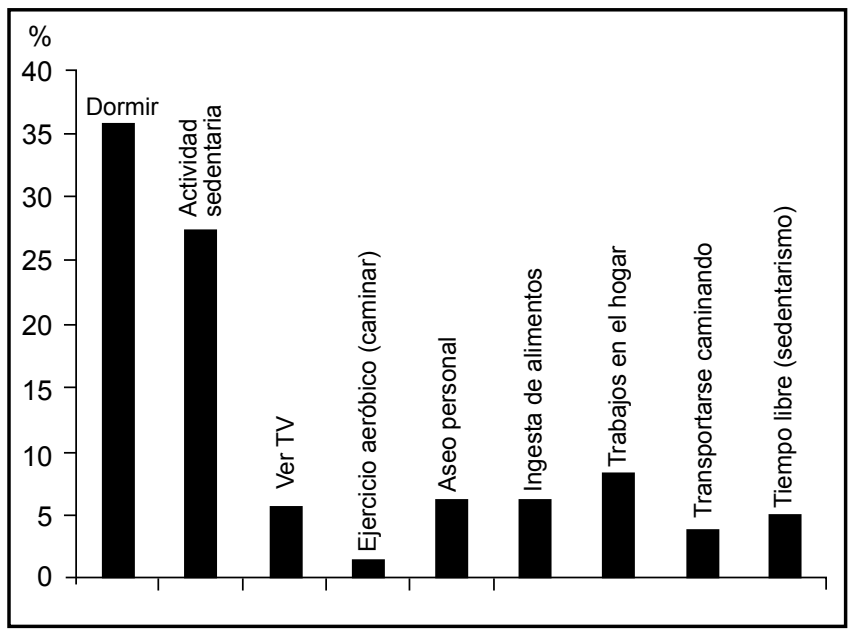

Figura 1. Distribución del tiempo semanal. 
de la paciente ( 8 hombres y 9 mujeres), 7 son diabéticos; ( 3 hombres y 4 mujeres, incluyendo a la paciente). Aún con estos antecedentes, la paciente no aprecia que el cambio en su estilo de vida pueda mejorar su condición, ya que posiblemente tenga afianzada la creencia de que las enfermedades se curan con medicinas y no modificando el estilo de vida, lo que involucra ingerir alimentos saludables y realizar regularmente actividad física. La paciente podría ubicarse en la etapa precontemplatoria, según el modelo de disposición motivada para el cambio por etapas ${ }^{6}$ en la que el sujeto no es activo físicamente y no piensa en modificar su comportamiento para convertirse en físicamente activo, al menos no en el grado (duración, frecuencia e intensidad) necesario para mejorar su estado mórbido.

El caso descrito puede reflejar una creencia arraigada en la sociedad costarricense. Los sujetos no son responsables cuando la salud les flaquea. En presencia de enfermedad acuden a los servicios de salud donde que el médico, mediante la prescripción de fármacos, cura la enfermedad. Los médicos prescriben medicamentos contra la enfermedad, pero en su repertorio no contemplan, al menos con la misma rigurosidad con que prescriben los medicamentos, cambios en el estilo de vida: actividad física y alimentación, como primer tratamiento. Los efectos operan en un mismo sentido: el paciente no considera que el estilo de vida, que está bajo su control, impactan su salud, y ni el médico, ni el sistema de educación y salud, consideran como la primera opción de tratamiento estos aspectos como preventivos y curativos. Es necesario trabajar en ambos aspectos para evitar que los costos de atención de la seguridad social se tornen inmanejables. ${ }^{14}$

Este caso sugiere que la gravedad del padecimiento de un sujeto tiene una influencia limitada sobre las medidas que este tome para modificar su estilo de vida. En la toma de acción para modificar el estilo de vida, que permitiría mejorar la calidad de esta, y la de sus próximos años y el costo para el sistema de salud, es determinante la percepción que tenga el sujeto sobre su enfermedad.

\section{Referencias}

1. El ejercicio físico ligero disminuye el riesgo de obesidad y diabetes tipo 2. Rev Panam Salud Pública 2003; 13: 333.

2. La actividad física y su efecto exclusivo sobre el riesgo de obesidad y diabetes. Rev Panam Salud Pública 2003; 14: 275-6.

3. ¿Puede la actividad física reducir el riesgo de accidentes cerebrovasculares? Rev Panam Salud Pública 2003; 14: 276.

4. Warburton DE, Nicol CW, Bredin SS. Health benefits of physical activity: the evidence. CMAJ 2006;174: 801-9.

5. Alberti KG, Zimmet P, Shaw J. International Diabetes Federation: a consensus on Type 2 diabetes prevention. Diabet Med 2007; 24: 45163.

6. American College of Sports Medicine. ACSM's Resource Manual for Guidelines for Exercise Testing and Prescription. 4th ed. Philadelphia: Lippincott Williams \& Wilkins; 2001.

7. Sáenz Madrigal M. Encuesta multinacional de diabetes mellitus, hipertensión arterial y factores de riesgo asociados. Área metropolitana San José [versión electrónica], 2004. Disponible en: http://www. ministeriodesalud.go.cr/Web\%20Direccion\%20Investigacion/ Archivos/enmetdia.pdf.

8. Brenes-Camacho G, Rosero-Bixby L. Diabetes mellitus en adultos mayores costarricenses. Población y Salud en Mesoamérica. Revista Electrónica; 2007; 5: artículo 2. Disponible en: http://ccp.ucr.ac.cr/ revista/volumenes/5/5-1/5-1-2/5-1-2.pdf.

9. Expert Panel on Detection, Evaluation, and Treatment of High Blood Cholesterol in Adults. Executive Summary of The Third Report of The National Cholesterol Education Program (NCEP) Expert Panel on Detection, Evaluation, And Treatment of High Blood Cholesterol In Adults [Adult Treatment Panel III (ATP-III)]. JAMA 2001; 285: 2486-97.

10. Friedewald WT, Levy RI, Fredrickson DS. Estimation of the concentration of low-density lipoprotein cholesterol in plasma, without use of the preparative ultracentrifuge. Clin Chem 1972; 18: 499-502.

11. Krentz AJ, Bailey CJ. Oral antidiabetic agents: current role in type 2 diabetes mellitus. Drugs 2005; 65:385-411.

12. Machado Alba JE, Moncada Escobar JC, Mesa Escobar G. Patrones de prescripción de antidiabéticos en un grupo de pacientes colombianos. Rev Panam Salud Pública 2007; 22: 124-31.

13. Organización Panamericana de la Salud. (2003). Informe final: IV taller CAMDI-Vigilancia y control de la diabetes en Centro América. Consultado en: http://www.paho.org/spanish/ad/dpc/nc/dia-camdi2003-inf-final.pdf

14. Ávalos A. Ticos consumen siete millones más de recetas. En: http:// www.nacion.com/ln_ee/2007/diciembre/19/pais1358768.html. 